PROCEEDINGS OF THE

AMERICAN MATHEMATICAL SOCIETY

Volume 130 , Number 12, Pages 3565-3570

S 0002-9939(02)06745-X

Article electronically published on July 2, 2002

\title{
CONTINUITY OF THE SUPPORT OF A STATE
}

\author{
ESTEBAN ANDRUCHOW
}

(Communicated by David R. Larson)

\begin{abstract}
Let $\mathcal{A}$ be a finite von Neumann algebra and $p \in \mathcal{A}$ a projection. It is well known that the map which assigns its support projection to a positive normal functional of $\mathcal{A}$ is not continuous. In this note it is shown that if one restricts to the set of positive normal functionals with support equivalent to a fixed $p$, endowed with the norm topology, and the set of projections of $\mathcal{A}$ is considered with the strong operator topology, then the support map is continuous. Moreover, it is shown that the support map defines a homotopy equivalence between these spaces. This fact together with previous work implies that, for example, the set of projections of the hyperfinite $\mathrm{II}_{1}$ factor, in the strong operator topology, has trivial homotopy groups of all orders $n \geq 1$.
\end{abstract}

\section{INTRODUCTION}

It is well known that the function which assigns its support projection $\operatorname{supp}(\varphi)$ to each positive normal functional $\varphi$ in a von Neumann algebra $\mathcal{A}$ is not continuous in any topology other than the coarse topology. This is the case even if $\mathcal{A}$ is finite dimensional. In fact, for any von Neumann algebra, it holds that the normal and faithful functionals are dense in norm among all the normal positive functionals (see [2]). However, a simple spectral argument shows that for matrix algebras, if one considers only functionals which have a priori equivalent supports, then the support function is continuous. The object of this note is to show that this holds for finite von Neumann algebras: i.e. the support is continuous among positive normal functionals with mutually Murray-von Neumann equivalent supports, when the set of projections of $\mathcal{A}$ is considered with the strong operator topology. Also, an easy example showing that this property does not hold for $B(H)$ is given.

Finally, again under the assumption that $\mathcal{A}$ is finite, it is shown that the map supp defines a homotopy equivalence between the space of states with support equivalent to $p$, in the norm topology, and the set of projections of $\mathcal{A}$ in the strong operator topology. Note that the connected components of the set of projections of a finite algebra coincide with the equivalence classes. In a previuos work with A. Varela [1 it is shown that this set of states has trivial homotopy groups of all orders for a class of factors which includes the hyperfinite $\mathrm{II}_{1}$ factor. It follows that for algebras in this class, the set of projections in the strong operator topology has trivial homotopy groups of all orders.

Received by the editors September 1, 2000.

2000 Mathematics Subject Classification. Primary 46L30, 46L05, 46L10.

Key words and phrases. State space, support projection.

(C)2002 American Mathematical Society 
Clearly it is sufficient to prove the continuity result for normalized functionals, i.e. states. For a fixed projection $p \in \mathcal{A}$, let $\Sigma_{p}(\mathcal{A})$ be the set of states of $\mathcal{A}$ with support equal to $p, P \Sigma_{p}(\mathcal{A})$ the set of states with support equivalent to $p$, and $\mathcal{S}_{p}(\mathcal{A})=\left\{x \in \mathcal{A}: x^{*} x=p\right\}$ the set of partial isometries of $\mathcal{A}$ with initial space $p$.

If $\psi$ is a state of $\mathcal{A}$ with support equivalent to $p$, then there exists a partial isometry $x \in \mathcal{S}_{p}(\mathcal{A})$ and $\varphi \in \Sigma_{p}(\mathcal{A})$ such that $\psi=\varphi\left(x^{*} \cdot x\right)$, that is, $\psi(a)=$ $\varphi\left(x^{*} a x\right)$. Namely, if $\operatorname{supp}(\psi)=q$ and $x$ is such that $x^{*} x=p$ and $x x^{*}=q$, put $\varphi(a)=\psi\left(x_{a x}^{*}\right)$. It is apparent that $\operatorname{supp}(\varphi)=p$ and $\varphi\left(x^{*} \cdot x\right)=\psi$. Conversely, note that if $\varphi \in \Sigma_{p}(\mathcal{A})$ and $x \in \mathcal{S}_{p}(\mathcal{A})$, then $\operatorname{supp}\left(\varphi\left(x^{*} \cdot x\right)\right)=x x^{*}$.

Note that $\Sigma_{p}(\mathcal{A})$ identifies with the set of faithful and normal states of $p \mathcal{A} p$. This algebra $p \mathcal{A} p$ will be considered in standard form, i.e. faithfully represented as operators on a standard Hilbert space $H$. As usual, $J$ will denote the antiunitary involution and $\mathcal{P} \subset H$ the positive cone. Recall that to every positive normal functional $\varphi$ of $p \mathcal{A} p$ there corresponds a unique vector $\xi \in \mathcal{P}$, such that $\xi$ induces $\varphi$, or $\varphi=\omega_{\xi}\left(\omega_{\xi}(a)=\langle a \xi, \xi\rangle\right.$, where $\langle$,$\left.\rangle denotes the inner product of H\right)$. States correspond to unit vectors, and faithful functionals correspond to cyclic and separating vectors (for $p \mathcal{A} p$ ). Moreover, the norm topology in the set of positive functionals is equivalent to the norm topology in $\mathcal{P}$ (see [2], 3]).

\section{Continuity of the Support}

The following lemma will be useful.

Lemma 2.1. Suppose that $y_{n}, x \in \mathcal{S}_{p}(\mathcal{A})$, and $\varphi_{n}, \varphi \in \Sigma_{p}(\mathcal{A})$, which are induced by vectors $\xi_{n}, \xi \in \mathcal{P}$. Then

$$
y_{n} \rightarrow x \text { strongly, and } \varphi_{n} \rightarrow \varphi \text { in norm }
$$

if and only if

$$
y_{n} \xi_{n} \rightarrow x \xi \text { in } H \text {. }
$$

Proof. If $\varphi_{n} \rightarrow \varphi$, then $\xi_{n} \rightarrow \xi$. Then $y_{n} \rightarrow x$ strongly and $\varphi_{n} \rightarrow \varphi$ in norm clearly imply that $y_{n} \xi_{n} \rightarrow x \xi$. On the other hand, suppose that $y_{n} \xi_{n} \rightarrow x \xi$. Then $x^{*} y_{n} \xi_{n} \rightarrow x^{*} x \xi=p \xi=\xi$. The normal functionals $\omega_{x^{*} y_{n} \xi_{n}}=\varphi_{n}\left(y_{n}^{*} x \cdot x^{*} y_{n}\right)$ converge to $\omega_{\xi}=\varphi$ in the norm topology of the conjugate space of $p \mathcal{A} p$. Consider the vectors $\gamma_{n}=x^{*} y_{n} J x^{*} y_{n} \xi_{n} \in \mathcal{P}$ (note that $\left.x^{*} y_{n} \in p \mathcal{A} p\right)$. Then

$$
\begin{gathered}
\omega_{\gamma_{n}}(a)-\varphi_{n}\left(y_{n}^{*} x a x^{*} y_{n}\right)=\left\langle a x^{*} y_{n} J x^{*} y_{n} J \xi_{n}, x^{*} y_{n} J x^{*} y_{n} J \xi_{n}\right\rangle-\left\langle a x^{*} y_{n} \xi_{n}, x^{*} y_{n} \xi_{n}\right\rangle \\
=\left\langle a x^{*} y_{n} \xi_{n}, x^{*} y_{n}\left(J y_{n}^{*} x x^{*} y_{n} \xi_{n}-\xi_{n}\right)\right\rangle=\left\langle a x^{*} y_{n} \xi_{n}, \Delta_{n}\right\rangle .
\end{gathered}
$$

Now $\left\|y_{n}^{*} x x^{*} y_{n} \xi_{n}-y_{n}^{*} x \xi\right\|=\left\|y_{n}^{*} x\left(x^{*} y_{n} \xi_{n}-\xi\right)\right\| \leq\left\|x^{*} y_{n} \xi_{n}-\xi\right\| \rightarrow 0$. Also note that $y_{n} \xi_{n} \rightarrow x \xi$ means that $\left\langle x^{*} y_{n} \xi_{n}, \xi\right\rangle \rightarrow 1$ (here we use the fact that $\left\langle y_{n} \xi_{n}, y_{n} \xi_{n}\right\rangle=$ $\langle x \xi, x \xi\rangle=1$ ), or equivalently $\left\langle\xi_{n}, y_{n}^{*} x \xi\right\rangle \rightarrow 1$, which by the same reason means that $\left\|\xi_{n}-y_{n}^{*} x \xi\right\| \rightarrow 0$. These two facts imply that $\Delta_{n} \rightarrow 0$. Therefore $\omega_{\gamma_{n}}-\varphi_{n}\left(y_{n}^{*} x \cdot x^{*} y_{n}\right)$ converges to zero in norm. It follows that $\omega_{\gamma_{n}} \rightarrow \varphi$ in norm, and therefore the vectors in $\mathcal{P}$ inducing these functionals converge in $H$, i.e. $\gamma_{n}=x^{*} y_{n} J x^{*} y_{n} \xi_{n} \rightarrow \xi$. Then

$$
\begin{aligned}
\left\|x^{*} y_{n} \xi-\xi\right\| & \leq\left\|x^{*} y_{n} J \xi-x^{*} y_{n} J x^{*} y_{n} \xi_{n}\right\|+\left\|x^{*} y_{n} J x^{*} y_{n} \xi_{n}-\xi\right\| \\
& \leq\left\|\xi-x^{*} y_{n} \xi_{n}\right\|+\left\|x^{*} y_{n} J x^{*} y_{n} \xi_{n}-\xi\right\|
\end{aligned}
$$

where both summands tend to zero by the remarks above. Therefore

$$
0 \leq\left\|y_{n} \xi-x \xi\right\|^{2}=\left\|y_{n} \xi\right\|^{2}+1-2 \operatorname{Re}\left\langle x^{*} y_{n} \xi, \xi\right\rangle \rightarrow 0
$$


(using that $\left\|y_{n} \xi\right\| \leq 1$ ). Since $\xi$ is separating for $p \mathcal{A} p$, it is cyclic for $(p \mathcal{A} p)^{\prime}=p \mathcal{A}^{\prime}$. Let $b^{\prime} \in p \mathcal{A}^{\prime}$; then $y_{n} b^{\prime} \xi=b^{\prime} p y_{n} \xi \rightarrow b^{\prime} p x \xi=x b^{\prime} \xi$. Since the vectors $b^{\prime} \xi$ are dense in $H$ and $y_{n}$ are bounded in norm, it follows that $y_{n} \eta \rightarrow x \eta$ for all $\eta$ in the range of $p(=H)$. Since the operators $y_{n}$ are all partial isometries with initial space $p$, it follows that $y_{n} \rightarrow x$ strongly. Also, we have proven that $y_{n}^{*} x \xi \rightarrow \xi$ and $y_{n}^{*} x \xi-\xi_{n} \rightarrow 0$. Then $\xi_{n} \rightarrow \xi$ in $H$, which are the vectors inducing the functionals $\varphi_{n}$ and $\varphi$. Therefore, $\varphi_{n} \rightarrow \varphi$ in norm.

Theorem 2.2. Suppose that $x_{n}, x \in \mathcal{S}_{p}(\mathcal{A})$ and $\varphi_{n}, \varphi \in \Sigma_{p}(\mathcal{A})$. Then

$$
\varphi_{n}\left(x_{n}^{*} \cdot x_{n}\right) \rightarrow \varphi\left(x^{*} \cdot x\right) \text { in the norm of the conjugate space of } \mathcal{A}
$$

if and only if there exist unitaries $u_{n} \in p \mathcal{A} p$ such that

$$
x_{n} u_{n} \rightarrow x \text { strongly, and } \varphi_{n} \circ \operatorname{Ad}\left(u_{n}\right) \rightarrow \varphi \text { in norm . }
$$

Proof. Note that $\varphi_{n} \circ \operatorname{Ad}\left(u_{n}\right)\left(\left(x_{n} u_{n}\right)^{*} \cdot x_{n} u_{n}\right)=\varphi_{n}\left(x_{n}^{*} \cdot x_{n}\right)$. If $y_{n}=x_{n} u_{n} \rightarrow x$ strongly and $\psi_{n}=\varphi_{n} \circ A d\left(u_{n}^{*}\right) \rightarrow \varphi$, then we must show that $\psi_{n}\left(y_{n}^{*} \cdot y_{n}\right) \rightarrow \varphi\left(x^{*} \cdot x\right)$. Note that

$$
\left\|\psi_{n}\left(y_{n}^{*} a y_{n}\right)-\varphi\left(x^{*} a x\right)\right\| \leq\left\|\psi_{n}\left(y_{n}^{*} a y_{n}\right)-\varphi\left(y_{n}^{*} a y_{n}\right)\right\|+\left\|\varphi\left(y_{n}^{*} a y_{n}\right)-\varphi\left(x^{*} a x\right)\right\| .
$$

The first summand is bounded by $\left\|\psi_{n}-\varphi\right\|\left\|y_{n}^{*} a y_{n}\right\| \leq\left\|\psi_{n}-\varphi\right\|\|a\|$. Suppose that $\varphi$ is induced by a vector $\eta$. Then $\varphi\left(y_{n}^{*} a y_{n}\right)=\left\langle y_{n}^{*} a y_{n} \eta, \eta\right\rangle=\left\langle a y_{n} \eta, y_{n} \eta\right\rangle$ and analogously $\varphi\left(x^{*} a x\right)=\langle a x \eta, x \eta\rangle$. Since $y_{n} \rightarrow x$ strongly, it follows that the functionals $\varphi\left(y_{n}^{*} \cdot y_{n}\right)$ converge to $\varphi\left(x^{*} \cdot x\right)$ in norm, a fact which deals with the second summand.

Suppose now that $\varphi_{n}\left(y_{n}^{*} \cdot y_{n}\right) \rightarrow \varphi\left(x^{*} \cdot x\right)$. Then

$$
\begin{aligned}
\left|\varphi_{n}(a)-\varphi\left(x^{*} x_{n} a x_{n}^{*} x\right)\right| & =\left|\varphi_{n}\left(x_{n}^{*} x_{n} a x_{n}^{*} x_{n}\right)-\varphi\left(x^{*} x_{n} a x_{n}^{*} x\right)\right| \\
& \leq\left\|\varphi_{n}\left(y_{n}^{*} \cdot y_{n}\right)-\varphi\left(x^{*} \cdot x\right)\right\|\|a\| .
\end{aligned}
$$

In particular, putting $a=p$, one has $\varphi\left(x^{*} x_{n} x_{n}^{*} x\right) \rightarrow 1$, i.e. $x^{*} x_{n} x_{n}^{*} x \xi \rightarrow \xi$ in $H$, where $\xi \in \mathcal{P}$ is the unique vector inducing $\varphi$. Indeed,

$$
\left\|x^{*} x_{n} x_{n}^{*} x \xi-\xi\right\|^{2}=\left\|x^{*} x_{n} x_{n}^{*} x \xi\right\|^{2}+1-2 \operatorname{Re}\left\langle x^{*} x_{n} x_{n}^{*} x \xi, \xi\right\rangle,
$$

and the claim follows using that $\left\|x^{*} x_{n} x_{n}^{*} x \xi\right\| \leq 1$ and $\left\langle x^{*} x_{n} x_{n}^{*} x \xi, \xi\right\rangle=\varphi\left(x^{*} x_{n} x_{n}^{*} x\right)$ $\rightarrow 1$. Therefore, for all $a \in p \mathcal{A} p$,

$$
\left\langle a x_{n}^{*} x \xi, x_{n}^{*} x \xi\right\rangle-\left\langle a x_{n}^{*} x J x_{n}^{*} x J \xi, x_{n}^{*} x J x_{n}^{*} x J \xi\right\rangle=\left\langle a x_{n}^{*} x \xi, x_{n}^{*} x J\left(\xi-x^{*} x_{n} x_{n}^{*} x \xi\right)\right\rangle \text {. }
$$

Since $x_{n}^{*} x J\left(\xi-x^{*} x_{n} x_{n}^{*} x \xi\right) \rightarrow 0$ in $H$, it follows that the induced functionals, i.e.

$$
\varphi\left(x^{*} x_{n} \cdot x_{n}^{*} x\right)-\omega_{x_{n}^{*} x J x_{n}^{*} x J \xi},
$$

tend to zero in norm. From the above remarks it follows that $\varphi_{n}-\omega_{x_{n}^{*} x J x_{n}^{*} x J \xi} \rightarrow 0$ in norm. Denote by $\xi_{n}$ the vectors in $\mathcal{P}$ inducing $\varphi_{n}$. Then

$$
x_{n}^{*} x J x_{n}^{*} x J \xi-\xi_{n} \rightarrow 0 .
$$

We claim that this implies that there exist unitaries in $p \mathcal{A} p$ such that $x_{n} u_{n} \rightarrow x$ strongly and $\varphi_{n} \circ \operatorname{Ad}\left(u_{n}\right) \rightarrow \varphi$ in norm. The vector in $\mathcal{P}$ inducing the functional $\varphi_{n} \circ \operatorname{Ad}\left(u_{n}\right)$ is $u_{n}^{*} J u_{n}^{*} J \xi_{n}$. By the above lemma, this claim is equivalent to saying that there exist unitaries such that

$$
x_{n} u_{n} u_{n}^{*} J u_{n}^{*} J \xi_{n}=x_{n} J u_{n}^{*} J \xi_{n} \rightarrow x \xi
$$

in $H$. Suppose that this is not the case. Then there would exist subsequences of $x_{n}$, $\xi_{n}$ (which we shall denote again by $x_{n}$ and $\xi_{n}$ ) such that for any choice of a unitary 
element $u$ in $p \mathcal{A} p$, one has $\left\|x_{n} J u^{*} J \xi_{n}-x \xi\right\| \geq d>0$. That is, $R e\left\langle x_{n} J u^{*} J \xi_{n}, x \xi\right\rangle \leq$ $1-d^{2} / 2$ for all unitaries $u$. Clearly this inequality is preserved by taking convex combinations of unitaries, as well as norm limits of these combinations. Therefore, by the Russo-Dye approximation theorem (see [3]), it follows that

$$
\operatorname{Re}\left\langle x_{n} J a \xi_{n}, x \xi\right\rangle \leq 1-d^{2} / 2
$$

for all $a \in p \mathcal{A} p,\|a\| \leq 1$. But this statement is in clear contradiction with the fact proved earlier that $x_{n}^{*} x J x_{n}^{*} x J \xi-\xi_{n} \rightarrow 0$ (choosing $a=x_{n}^{*} x$ for appropriate $n$ ).

Now our main result follows.

Theorem 2.3. Let $\Phi_{n}$ be states of a finite von Neumann algebra $\mathcal{A}$, with support projection equivalent to a given projection $p$, such that $\Phi_{n} \rightarrow \Phi$ in norm, and the support of $\Phi$ is also equivalent to $p$. Then $\operatorname{supp}\left(\Phi_{n}\right) \rightarrow \operatorname{supp}(\Phi)$ strongly.

Proof. It was shown that states with support equivalent to a given projection are of the form $\Phi_{n}=\varphi_{n}\left(x_{n}^{*} \cdot x_{n}\right), \Phi=\varphi\left(x^{*} \cdot x\right)$, where $\operatorname{supp}\left(\varphi_{n}\right)=\operatorname{supp}(\varphi)=p$ and $x_{n}, x \in \mathcal{S}_{p}(\mathcal{A})$. By the theorem above, there exist unitaries $u_{n}$ in $p \mathcal{A} p$ such that $x_{n} u_{n} \rightarrow x$ strongly. Since $\mathcal{A}$ is finite, this implies that $u_{n}^{*} x_{n}^{*} \rightarrow x^{*}$. Also, since these sequences are bounded in norm, the sequence of the products is also strongly convergent. Therefore $x_{n} u_{n} u_{n}^{*} x_{n}^{*}=x_{n} x_{n}^{*} \rightarrow x x^{*}$ strongly. But as it was shown before, $\operatorname{supp}\left(\varphi_{n}\left(x_{n}^{*} \cdot x_{n}\right)\right)=x_{n} x_{n}^{*}$ and $\operatorname{supp}\left(\varphi\left(x^{*} \cdot x\right)\right)=x x^{*}$, which completes the proof.

It is not difficult to find an example of a sequence of faithful normal states in $B(H)$, which converge in norm to a nonfaithful state, with support an infinite rank projection (therefore equivalent to the identity). In other words, the result above is not valid for infinite algebras. The following example is taken from [1.

Example 2.4. Let $a \in B\left(\ell^{2}(\mathbb{N})\right)$ be a trace class positive diagonal operator with trace one, and no zero entries in the diagonal. Put $\varphi(x)=\operatorname{Tr}(a x), x \in B\left(\ell^{2}(\mathbb{N})\right)$. Clearly, $\varphi$ is faithful. Let $b$ be the unilateral shift in $\ell^{2}(\mathbb{N})$. Denote by $q_{n}$ the $n \times n$ Jordan nilpotent, and by $w_{n}$ the unitary operator on $\ell^{2}(\mathbb{N})$ having the unitary matrix $q_{n}+q_{n}^{* n-1}$ on the first $n \times n$ corner and the rest of the diagonal completed with 1 . Then $\varphi\left(w_{n}^{*} \cdot w_{n}\right)$ is a faithful state, $\operatorname{supp}\left(\varphi\left(b^{*} \cdot b\right)\right)=b b^{*} \neq 1$, and all these states have equivalent supports. We claim that $\varphi\left(w_{n}^{*} \cdot w_{n}\right)$ converge to $\varphi\left(b^{*} \cdot b\right)$ in the norm topology. Indeed

$$
\begin{aligned}
& \left|\varphi\left(w_{n}^{*} t w_{n}\right)-\varphi\left(b^{*} t b\right)\right|=\left|\operatorname{Tr}\left(a\left(w_{n}^{*} t w_{n}-b^{*} t b\right)\right)\right| \\
& \leq\left|\operatorname{Tr}\left(a\left(w_{n}^{*}\left(t w_{n}-t b\right)\right)\right)\right|+\left|\operatorname{Tr}\left(a\left(w_{n}^{*}-b^{*}\right) t b\right)\right| .
\end{aligned}
$$

By the Cauchy-Schwarz inequality, the first summand can be bounded by

$$
\|t\| \operatorname{Tr}\left(a\left(2-w_{n}^{*} b-b^{*} w_{n}\right)\right) .
$$

Since $\operatorname{Tr}(a)=1$, this term equals

$$
\|t\|\left(2-\operatorname{Tr}\left(a w_{n}^{*} b+a b^{*} w_{n}\right)\right)=2\|t\| \sum_{k \geq n} a_{k},
$$

where $a_{k}$ are the diagonal entries of $a$. It is clear that this term tends to zero when $n \rightarrow \infty$. The other summand can be dealt with in a similar way, establishing our claim. 


\section{HOMOTOPY PROPERTIES OF THE SUPPORT}

From now on we will suppose that $\mathcal{A}$ is a finite von Neumann algebra. Fix a faithful normal tracial state $\tau$. Using $\tau$ it is easy to find a copy of the set $\mathcal{E}_{p}$ of projections on $\mathcal{A}$ which are equivalent to $p$, which in this case coincides with the unitary orbit of $p$, inside the set of states with support equivalent to $p$; namely, as the set $\left\{\tau_{q}: q \in \mathcal{E}_{p}\right\}$ where $\tau_{q}$ denotes the state $\tau_{q}(a)=\tau(q a) / \tau(p)$ (note that $\tau(p)=\tau(q))$. Moreover, this formula defines a continuous section of supp. Let us denote by $P \Sigma_{p}(\mathcal{A})$ the set of states of $\mathcal{A}$ with support equivalent to $p$.

Proposition 3.1. The map $\mathcal{E}_{p} \rightarrow P \Sigma_{p}(\mathcal{A}), q \mapsto \tau_{q}$ is a continuous section for supp. The set $\mathcal{E}_{p}$ with the strong operator topology is homeomorphic to the image $\left\{\tau_{q}: q \in \mathcal{E}_{p}\right\}$ of this section, regarded with the norm topology (of the conjugate space of $\mathcal{A})$.

Proof. It is clear that the support of $\tau_{q}$ is $q$, therefore this map is a section. Let us see that it is continuous. Let $p_{n}$ converge strongly to $q$ in $\mathcal{E}_{p}$. Suppose $\mathcal{A}$ is represented in a Hilbert space $H$ in such a way that $\tau$ is given by a (tracial) vector $\nu$. For instance, take $H=L^{2}(\mathcal{A}, \tau)$. Then

$$
\left|\tau\left(p_{n} a\right)-\tau(q a)\right|=\left|\left\langle\left(p_{n}-q\right) a \nu, \nu\right\rangle\right|=\left|\left\langle a \nu,\left(p_{n}-q\right) \nu\right\rangle\right| \leq\|a\|\left\|\left(p_{n}-q\right) \nu\right\| .
$$

Dividing by the common trace of all these projections, one obtains that $\tau_{p_{n}} \rightarrow \tau_{q}$ in norm. It remains to prove the converse, that if $\tau_{p_{n}} \rightarrow \tau_{q}$ in norm for $p_{n}, q \in \mathcal{E}_{p}$, then $p_{n} \rightarrow q$ strongly. Since $H=L^{2}(\mathcal{A}, \tau)$ is a standard form for $\mathcal{A}$, convergence in norm of the positive functionals $\tau_{p_{n}}$ means convergence in $H$ of their densities $p_{n} \nu$. It follows that $p_{n} \nu \rightarrow q \nu$. Let $a^{\prime}$ be an element in the commutant of $\mathcal{A}$. Then $a^{\prime} p_{n} \nu=p_{n} a^{\prime} \nu \rightarrow q a^{\prime} \nu$. Therefore $p_{n}$ converges to $q$ in a dense subset of $H$, namely $\left\{a^{\prime} \nu: a^{\prime} \in \mathcal{A}^{\prime}\right\}$. Since $p_{n}, q$ have norm 1 , it follows that $p_{n} \rightarrow q$ strongly.

In particular this implies that supp is an open mapping in our context.

The fibre of the map supp over each projection in $P \Sigma_{p}(\mathcal{A})$ is convex. This enables one to prove that in fact $\mathcal{E}_{p}$ is a strong deformation retract of $P \Sigma_{p}(\mathcal{A})$.

Proposition 3.2. The image $\left\{\tau_{q}: q \in \mathcal{E}_{p}\right\}$ of the cross-section above is a strong deformation retract of $P \Sigma_{p}(\mathcal{A})$.

Proof. Consider the continuous map $F_{t}(\Phi)$ given by

$$
F_{t}(\Phi)=t \tau_{\operatorname{supp}(\Phi)}+(1-t) \Phi
$$

for $\Phi \in P \Sigma_{p}(\mathcal{A})$ and $t \in[0,1]$. Then $F_{0}=I d, F_{1}$ is a retraction onto the image of the cross-section of supp (that is, essentially $F_{1}=\operatorname{supp}$ ), and for all $t \in[0,1]$, $F_{t}\left(\tau_{q}\right)=\tau_{q}$

This implies that the space $P \Sigma_{p}(\mathcal{A})$ in the norm topology has the same homotopy type as the space of projections of $\mathcal{A}$ in the strong operator topology.

In 4] S. Popa and M. Takesaki considered $\mathrm{II}_{1}$ factors $\mathcal{R}$ which when tensored with an infinite type I factor admit a one-parameter group of automorphisms which scales the trace. They proved that the unitary group of such factors is contractible in the strong operator topology. This class of algebras includes among others the hyperfinite factor $\mathcal{R}_{0}$. Using their results and techniques, it was proven in [1] that the set $P \Sigma_{p}(\mathcal{R})$ in the norm topology has trivial homotopy groups of all orders. The following result follows. 
Corollary 3.3. Let $\mathcal{R}$ be a $I I_{1}$ factor with the property above. Let $p \in \mathcal{R}$ be a projection. Then the set $\mathcal{E}_{p}$ of projections of $\mathcal{R}$ which are equivalent to $p$, regarded with the strong operator topology, has trivial homotopy groups of all orders.

\section{REFERENCES}

[1] E. Andruchow, A. Varela, Homotopy of vector states, preprint.

[2] O. Bratteli, D.W. Robinson, Operator algebras and quantum statistical mechanics, vol. I, Springer, Berlin, 1981. MR 81a:46070

[3] R.V. Kadison and J.R. Ringrose, Fundamentals of the theory of operator algebras, Vol. II, Academic Press, New York, 1986. MR 88d:46106

[4] S. Popa, M. Takesaki, The topological structure of the unitary and automorphism groups of a factor, Commun. Math. Phys. 155 (1993), 93-101. MR 94h:46092

Instituto de Ciencias, Universidad Nacional de Gral. Sarmiento, J. M. Gutierrez entre J.L. Suarez y Verdi, (1613) Los Polvorines, Argentina

E-mail address: eandruch@ungs.edu.ar 